\title{
Using Concepts Maps in a Foundation Mathematics Course: What Have we Learnt?
}

\author{
Sali Hammad ${ }^{1 *}$, Christos Dimitriadis $^{2}$, Ted Graham $^{3}$ \\ ${ }^{1}$ Gulf University for Science and Technology, KUWAIT \\ 2 Kingston University, UK \\ ${ }^{3}$ Independent Researcher, UK
}

Received 9 December 2020 - Accepted 22 January 2021

\begin{abstract}
This paper reports on the findings from the use of concept maps in a mathematics foundation course at a university in Kuwait. The study sample consisted of 130 freshmen students from a mathematics foundation course. Through a case-study design, concept maps and assessment tests were utilized to assess and monitor students' mathematical understanding and achievement at various points of the course. The findings of the study showed that concept maps can be effective as a tool to assess, monitor, and improve students' mathematical understanding, particularly their conceptual understanding when they are used systematically and when their use is followed-up by discussions that encourage students to reflect and talk about their maps and the links they have made. The improved understanding was found to contribute to the enhancement of mathematical achievement. The paper reports details on the use of concept maps in mathematics lessons and makes recommendations for practice and future research.
\end{abstract}

Keywords: mathematical understanding, concept maps, procedural understanding, conceptual understanding

\section{INTRODUCTION}

Students have scored in the lower percentiles during the past five years at some public and private mathematics university entrance exams in Kuwait (Soliman \& Hilal, 2016). Soliman and Hilal (2016) stressed the weak mathematical knowledge that students have had when applying for the Kuwait University entrance exams that showed only a $26 \%$ success rate. Similar success rates have appeared in the institution Gulf University for Science and Technology (GUST), where this study took place. The university's statistics for the past five academic years revealed that an average of only $26 \%$ of the total number of students applying passed the university entrance exam and proceeded directly to academic courses. The other $74 \%$ of the students were weaker in their mathematical knowledge and were required to take the mathematics foundation courses at the Mathematics Foundation Unit (MFU). These low achieving students were required to take various foundation mathematics courses depending on their entrance exam results to improve their background information in Arithmetic and Algebra.

Through this study, the authors wanted to find out whether and how we could support those students fill in the gaps in their knowledge and enhance their mathematics achievement by improving their understanding of mathematical concepts we were teaching them. The study specifically focuses on the use of concept maps to identify weaknesses in mathematical learning and to support and monitor the development of conceptual understanding.

\section{LITERATURE REVIEW}

Michener (1978) defined mathematical understanding as the connection between different knowledge resources and items which also involves the learners' awareness of the purposes of using different types of mathematical skills and techniques. Researchers (e.g., Baroody, 2003; DeCaro, 2016; Heinze et al., 2009; Schneider et al., 2011; Siegler, 1994; Verschaffel et al., 2009) clarified that the knowledge of a variety of solution 


\section{Contribution to the literature}

- This study provides evidence from the use of concept maps in a mathematics classroom to improve students' conceptual understanding and mathematical competence.

- It provides examples from the use of concept maps for assessing and monitoring students' mathematical understanding.

- It also highlights the importance of reflection and metacognitive discussions among the students around their concept maps, and the teacher's role in actualizing the benefits of using concept maps in mathematics learning and teaching environment.

strategies reflects a deeper conceptual understanding for the learners. Also, knowledge can be complex, varied, constructed, and can be structured as complicated conceptions (Reinfried, 2006).

Wilkerson-Jerde and Wilensky (2011) linked the development of mathematical understanding with the development of networks and connections. They recommended that these networks and connections should involve: (1) Mathematical knowledge as a network: relating the nature of mathematical understanding to the structure of mathematical knowledge as a network of relations between different properties, objects, and procedures for the mathematical idea. (2) The role of different resources in learning: the resources of knowledge network can vary in type and purpose. They can include images, experiences, specific features, and any other knowledge associated with the name of a mathematical concept, facts, algorithms, routine procedures, the role of examples (specifically, those generated by the learner), the generic properties of mathematical ideas, visual resources in mathematical practice, and combine the use of strategic and types of resources together. (3) Learning as building connections: mathematical understanding is about having, building, and enhancing knowledge by linking the use between resources and connections. This means that, educators can combine different lecture-styles of teaching with teaching resources to improve students' mathematics conceptual understanding. The lecture-styles of teaching can include the use of concept maps as it involves "a diagram representing the conceptual structure of a subject discipline as a graph in which nodes represent concepts and connections represent cognitive links between them" (McGowen \& Tall, 1999, p. 2).

Concept mapping is used to outline relationships between concepts and ideas but it cannot be used to connect spontaneous elements, which makes it a relational device (Davies, 2011). Concept mapping has been used in science and mathematics education by different researchers (e.g., Cliburn, 1990; Lambiotte \& Dansereau, 1991; Lanier, 1997; Laturno, 1993; McGowen \& Davis, 2019; McGowen \& Tall, 1999; Moreira, 1979; Novak, 1990; Park \& Travers, 1996; Skemp, 1986; Williams, 1998). They have found that concept mapping can facilitate students' learning into meaningful learning by helping them develop cognitive and metacognitive learning skills, which will eventually enhance their academic success and achievement. They also explained that teachers can use concept maps as an important tool to identify students' weaknesses and focus on their needs in the learning process. This means, the use of concept maps can be significantly effective to explore and improve students' educational understanding which can be used as a mechanism in the mathematics classroom at the college level. McGowen and Tall (1999) used concept maps to monitor students' cognitive development during a mathematics course and study the qualitative differences between students of different levels of achievement. They found that a low achieving student was unable to maintain stable connections between the objectives when drawing a sequence of concept maps. The student showed confusion when asked to solve a new problem that required the application of previous knowledge in unfamiliar situations as she was trying to recall specific methods or steps of solutions previously learnt. In contrast, a high achieving student could relate new knowledge to ideas that had been previously acquired, building on the cognitive collage he hadalready constructed. He was able to create stable connections between the elements of his maps and built more of a conceptual structure in his understanding (McGowen \& Tall, 1999). The structure of every student's map is considered unique to its author's learning experiences, knowledge, and understanding of each topic and reflects the author's thinking construction not just their memory (Jonassen et al., 1997). As such, concept maps can be seen to have the potential to be a powerful tool for teachers to assess students' understanding and their way of thinking, identify particular strengths or weaknesses and needs, and promote meaningful learning.

Skemp (1976) was one of the first researchers who discussed "understanding" in mathematics and divided it into two types: relational and instrumental understanding. The former is about "knowing both what to do and why" (Skemp, 1976, p. 2) while the latter is about following "rules without reasons" (Skemp, 1976, p. 2). The latter is often quicker, as it does not require time for explanations, and when it is grasped by the students adequately, it leads to correct responses and immediate rewards. It is similar to "procedural" understanding as suggested by other experts in the field 
(Gray \& Tall, 1994; Hiebert \& Lefevre, 1986; Hiebert \& Carpenter, 1992; Kadijevich \& Haapasalo, 2001; Peled \& Segails, 2005; Sfard, 1991), and it heavily relies on a short memory. On the other hand, relational understanding requires more time to explain a concept, how it might relate to different concepts and why, similarly to "conceptual" understanding recommended by other more recent authors (Gray \& Tall, 1994; Hiebert \& Carpenter, 1992; Hiebert \& Lefevre, 1986; Kadijevich \& Haapasalo, 2001; Miller \& Hudson, 2007; Peled \& Segails, 2005; Rittle-Johnsons \& Alabali's, 1999; Sfard, 1991; Tall, 1994b). As it is based on a deep understanding of the concept and possible relationships, relational (and conceptual) understanding is more 'transferable and applicable' to new tasks. It does not rely on a short memory and is more effective in the long term. It is, according to Skemp (1976), the real understanding needed for mathematics students.

Conceptual understanding is often linked to the 'constructivist' view of learning. Von Glasersfeld (1998) referred to constructivism in psychology as 'trivial constructivism' which is based on the idea that knowledge is not received but is actively built up by the cognizing subject (Ernest, 1991). Different constructivists such as (Kelly, 1955; Piaget \& Cook, 1952; Rumelhart \& Norman, 1978; Skemp, 1979) agree that individual knowledge development is based on the construction of mental structures such as concepts and schemata and that for this experience and reflection play a crucial role (Ernest, 1991). To the latter, Ernest (2011) also adds the role of interaction with others (peers and the teacher) in the construction and re-construction of knowledge drawing upon social constructivist ideas (e.g. Vygotsky, 1978). Both cognitive constructivist (e.g. Piaget) and social constructivist (e.g. Vygotsky) ideas are recommended for teaching as part of pedagogical constructivism (Driver, 1981; Kinchin et al., 2000; Pfund \& Duit, 1994; Reinfried, 2006). This might empower teachers to develop some innovative teaching methods which create the awareness to focus on the learners' needs to reach a deeper level of understanding. This also links with the ideas that we discussed at the beginning of this literature review regarding the importance of building connections and networks for the development of mathematical understanding, which is supported by Hiebert and Lefevre (1986), and Miller and Hudson (2007) who explained that conceptual knowledge is a connected piece of information when the learner recognizes its relationship to other pieces of information. This means constructing relationships between the pieces of a wide range of knowledge leads to the development of conceptual knowledge and understanding.

There are some disadvantages that some researchers have discussed when using concept maps (Davies, 2011) such as 1) they can be idiosyncratic in terms of design, 2) because of their complexity they may not always assist memorability, 3) learners faced with designing concepts maps often feeling overwhelmed and de-motivated (Davies, 2011, p. 285). The authors discuss how they could overcome those disadvantages later in the study.

\section{RESEARCH METHODOLOGY}

\section{Sampling and Participation}

The researcher used a case study methodology in order to focus on individual students' changes in their mathematics understanding, particularly conceptual understanding and achievement. Using the case study approach could provide detailed analysis such as using the researcher's own diary notes and process-tracking. We followed guidance from Thomas (2011) to clarify the case and set boundaries for the case study by establishing the "subject" (the case itself) and the "object" of the study (the frame or theory which the subject illustrates and explicates). The subject of our study was a group of students at a foundation course in one university trying to improve their mathematical knowledge and understanding to succeed in entry exams. The object (which, according to Thomas, should be linked with a theoretical basis) was the possible impact of the inclusion of concept maps that encourage higher-order thinking and conceptual understanding in mathematics learning.

In order to grant ethics for the study, the lead researcher received an 'Institutional Review Board' (IRB) clearance from GUST in order to conduct the study. Also, she received approval from the MFU director. The participating students could keep a copy of the research information sheet that was provided for them. This included an explanation of the purpose of the study and offered the right to withdraw, the rights and obligations to confidentiality, and provided opportunities to allow participants to ask questions about any part of the research as recommended by Cohen et al. (2013). The study was applied in two academic semesters with students who were studying the Intermediate Algebra (Math096) course. The first data collection was completed during Spring 2017. It included the groups 1 to 4 . The second data collection was completed during the Fall 2018 and it consisted of groups 5 to 8 . The groups of students included a total number of 72 male and 58 female students to make a total of 130 students. Also, all members of the groups of students were in the age range of 18-32 years old. All the groups were provided with the Research Information Sheet which explained the focus of the study. Also, they were asked to participate in the study voluntarily without feeling any pressure by their student-teacher relation. This and more was included in the Research Information Sheet and Consent Form. The participating groups were assigned to the lead researcher as regular teaching classes and teaching load by the university registrar's office, MFU director, and coordinator. 


\section{The Scheme of the Study}

The study was divided into four stages, as follows:

- Stage 0 (the first day or two of the course): The students drew the first individual concept maps connecting basic Arithmetic and Algebraic objectives. After submitting their concept maps, the teacher photocopied the maps and gave them back to the students. She created discussion groups among the students and asked them to talk about their maps and explain their thinking. The aim was to create conditions to engage students in higher-order thinking. During the discussion, the teacher was displaying anonymous examples from students' work and asking questions to encourage them think and talk (see details in the following section). In addition, the students completed a baseline level quiz on basic algebraic and arithmetic questions as shown in Appendix A.

- Stage 1 (After the first month of the course): The students completed Test 1 . The teacher continued encouraging the students to think and talk about their completed concept maps, using group discussions and demonstrations on the board.

- Stage 2 (Midcourse-second month of the course): The students completed Test 2 and their second individual concept maps. The teacher continued encouraging the students to think and talk about their completed concept maps, using group discussions and displays of concept maps on the board.

- Stage 3 (Last week of the course): The students completed Test 3 (The final examination) and the third individual concept maps. The course ended with semi-structured interviews with students who volunteered to complete the interview with the teacher. The same interview questions were used for all the participating students.

For the periods between stages, the researcher used different metacognitive and higher order thinking practices as explained in the following section.

\section{Instruments Used in the Study}

\section{Concept maps: Major instrument}

Three hundred and ninety concept maps were collected at three stages during the course. The students had never used concept maps before and were not familiar with them at the start of the course. The researcher shared one simple science example with the students at the start of the course and explained the relations between different concepts and how to connect them in a concept map. The science example was used to avoid providing them with mathematical examples of concept maps. This would help the students to understand what a concept map is and how it can be created but will not allow them to copy mathematical ideas. The researcher also explained some methods of concept map designs demonstrating different structural typologies, such as those identified by Kinchin et al. (2000) as 'spoke', 'chain', and 'net'. Spoke refers to a structure with a single core concept and some subordinate concepts linked with the core through simple direct links but not with each other (there are no cross-links between the concepts). Chain refers to a structure with a linear sequence, where each concept is linked only to those preceding and following, indicating a logical sequence and hierarchical structure but lacking cross-linking. Net refers to a highly integrated structure with cross-linking between several concepts and indicates holistic learning and deep understanding (the more complex the links the more holistic and deeper the understanding). Figure 1 illustrates the structural differences between the spoke (A), chain (B), and net (C) (2000, p. 47). The students then were asked to create their own concept maps for the mathematical objectives that they had been taught. The students were not allowed to communicate with each other while drawing their individual concept maps. After submitting their concept maps, students were allowed to discuss how they connected those objectives with each other in groups. Data from students' concept maps were collected at the start, middle, and end of course. For the periods between the stages, the researcher used different concept map samples one or two times a week to create group discussions and engage students in higher-order thinking. The latter was achieved by encouraging students to reflect on and talk about their own thinking (metacognition), understanding or misconception points of different links presented in the maps. For example, the teacher would present one anonymous example using the in-class projector, and encouraged the students to think and explain why did the sample student think to connect any certain concept to another? Could he/she connect it differently? What were his/her other options of connectivity? Why would other options be better or worse? What was he/she thinking about when decided to connect them this way? Why did he/she reject other options? What made him/her change opinion? During the course, the researcher aimed to maintain discussions that promoted students' higher-order thinking through their concept maps explorations. She kept her own diary observations for the purpose of keeping her personal thoughts and any changes in her plans. Also, she used it for her reflections to address biases related to positionality and personal beliefs/expectations.

The maps were analyzed qualitatively in order to reflect students' mathematical-conceptual understanding, if any, during the course and quantitatively to determine connectivity levels within each map. Informed by previous similar studies (e.g. Kinchin et al., 2000; McGowen \& Tall, 1999), this study developed a 


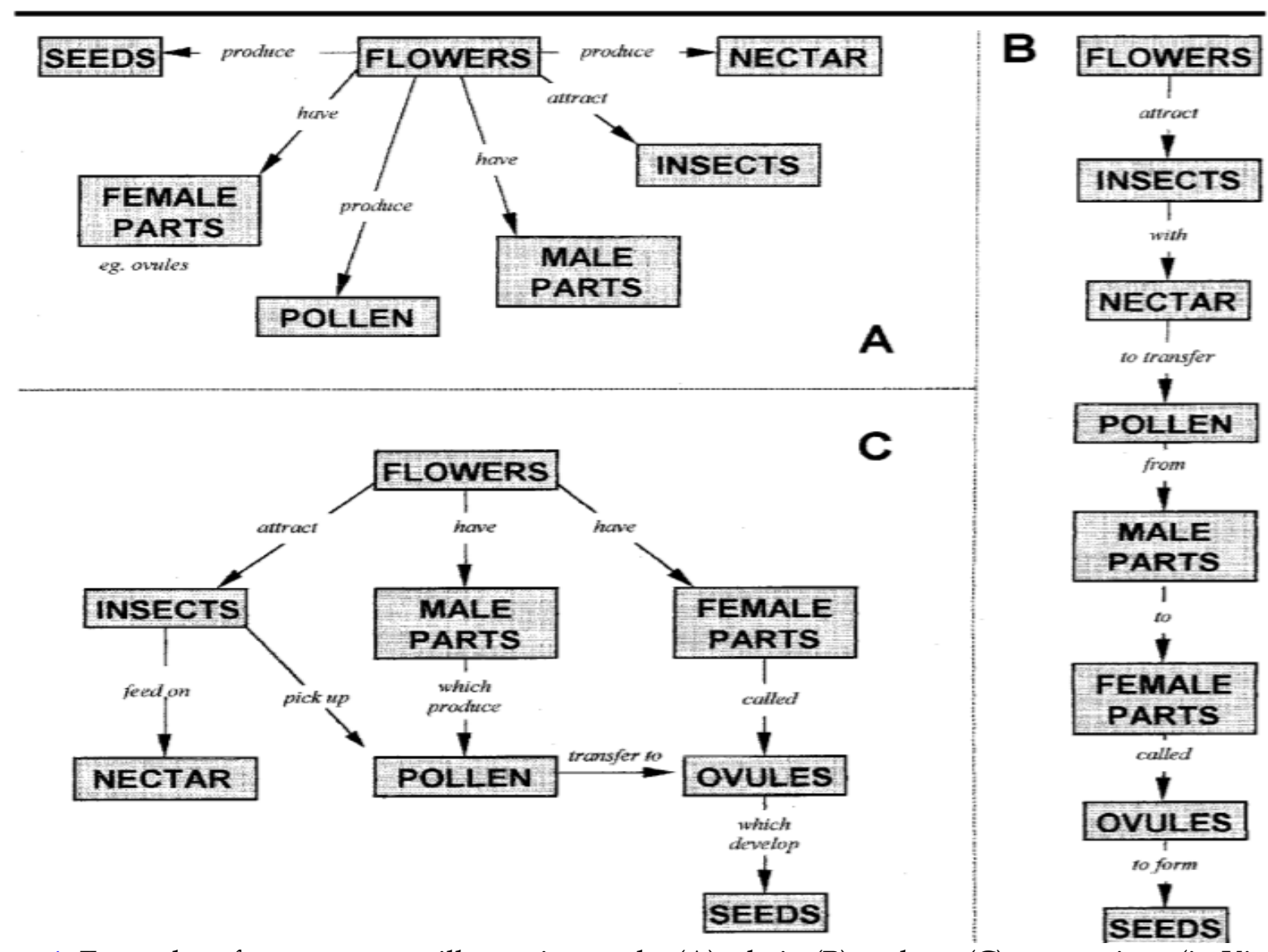

Figure 1. Examples of concept maps illustrating spoke (A), chain (B) and net (C) connections (in Kinchin et al. 2000, p 47)

simple technique for the quantitative analysis of concept maps, mainly based on Kinchin et al.'s (2000) method for calculating valid and invalid links. This involved a coding system from 1 (low connectivity) to 3 (high connectivity) to determine the level of connectivity based on the number of valid links (see Table 2) assuming that high connectivity is an indicator of a more conceptual understanding. The connectivity code was calculated for each concept map using the formula:

Score $=$ Number of valid links - Number of invalid links

(see examples in Figures 2, 3, \& 4). The coding system was used to produce numbers for quantitative analysis of students' connectivity progression during the course.

\section{Level quiz and test scores' progression: Supporting instruments}

The purpose of using the level quiz was to explore the students' background knowledge in mathematics, especially in basic Algebra and Arithmetic. The level quiz and other assessments used in the course included questions that can be analyzed procedurally or conceptually in order to explore students' understanding. Appendix A shows the questions used in the level quiz. Table 1 presents the kind of questions used categorized as 'procedural' or 'conceptual' according to the type of solving process and understanding required.
A question is considered conceptual if it is based on devising a strategy(s) or technique(s) in order to answer the question rather than simply recalling a method. Also, conceptual questions are higher-order thinking questions. An example of a conceptual question is

"Solve and Graph: $-19 \leq-2 x-7$ and $-7-2 x<-20$ "

where the students have to solve each inequality separately, graph the solutions on one number line and devise a technique in order to translate 'and' into a solution using interval notation. It is easy for the students to misunderstand the meaning of 'and' or 'or' on the number line. This is not typical of a question that students have studied in class. Other examples of a conceptual questions can be questions 4 or 5 shown in the level quiz shown in Table 1 and in Appendix A. A question is considered procedural if it is based on recalling familiar strategies. An example of a procedural question is

$$
\text { "Solve: } 10-3(2 x-1)-5=1-5 \text { " }
$$

This is a typical question that students have practiced in-class sessions and using an online homework program. Other examples of procedural questions can be seen in Table 1 and Appendix A. The scores from the level quiz and all tests were collected and analyzed through the use of SPSS in order to analyses students' progression if any. 
Table 1. The procedural and conceptual description of each question in the level quiz

\begin{tabular}{|c|c|c|}
\hline Question number and type & Style & Description \\
\hline 1-a) Simplify BEDMAS & Procedural & Use BEDMAS properties to simplify the question. \\
\hline b) Simplify using exponential properties & Procedural & Use exponential properties to simplify the expression. \\
\hline 2-a) Arithmetic & Conceptual & $\begin{array}{l}\text { Demonstrate an understanding of the issues of the concept of } x / 0 \\
\text { when substituting } x \text { by a given value }\end{array}$ \\
\hline b) Arithmetic & Conceptual & $\begin{array}{l}\text { Demonstrate an understanding of the issues of the concept of } 0 / x \\
\text { when substituting } x \text { by a given value }\end{array}$ \\
\hline 3- Solve Linear equation with one variable & Procedural & Use algebraic properties to find the value of the unknown. \\
\hline 4- Word problem (\%). & Conceptual & $\begin{array}{l}\text { Translate a context described in words into an algebraic equation } \\
\text { (using percentage) and solve for the unknown. }\end{array}$ \\
\hline $\begin{array}{l}5 \text { - Solving algebraic equation using } \\
\text { operations on polynomials. }\end{array}$ & Conceptual & $\begin{array}{l}\text { Translate the meaning of equilibrium into an equation (Revenue = } \\
\text { Cost). Use the appropriate technique to solve the algebraic equation } \\
\text { in order to find the number of cellphones produced. }\end{array}$ \\
\hline
\end{tabular}

Table 1 shows the procedural and conceptual description of each question in the level quiz shown in Appendix A.

To examine the students' performance over the course and see whether there was any change considering procedural or conceptual understanding, similar questions between the level quiz and Test 3 were examined. The study compared the success rate for the three similar questions between the level quiz and Test 3 . The aim of using this comparison was to determine whether students improved their procedural and/or conceptual understanding from the level quiz to Test 3.

\section{Interviews: Supporting instruments}

Twenty-five students were interviewed using semistructured interviews at the end of the course. The students were selected based on their willingness to be interviewed. The 25 students who volunteered were divided into three categories according to their accumulative overall grades towards the end of the course; high, middle, and low achieving students based on their cumulative grades. These students were asked to discuss and explain the differences between their concept maps across the course, why, and how it changed from one stage to another. The interviews were audio recorded, transcribed, coded and turned into themes. Students expressed their opinions about the use of concept maps in the classroom and their mathematical understanding progress during the course.

\section{Research journal: Supporting instruments}

A research journal was used to keep track of the daily activities and behaviors, note changes, occurring events in the class, and capture the researcher's self-reflections. A research journal may be subjective and biased because what is recorded and interpreted relies on the researcher. However, if the principles of reflexivity in research (Berger, 2015) are adopted, researchers' bias and subjectivity can be overcome. The journal itself can become a tool in supporting the researcher's reflexivity, e.g., when the researcher reflects on the questions asked in a session or their approach that might have
Table 2. Concept maps' connectivity codes and their scores

\begin{tabular}{lcc}
\hline Name of Code & Code Number & Score \\
\hline Low Connectivity & 1 & $0-15$ \\
Medium Connectivity & 2 & $16-22$ \\
High Connectivity & 3 & More than 22 \\
\hline
\end{tabular}

encouraged a particular reaction and realizes leading questions or biased approach and then makes changes to address the problems.

\section{RESULTS AND DATA ANALYSIS}

\section{Concept Maps; Qualitatively and Quantitatively; Major Results}

Table 2 shows the concept maps' connectivity codes used in the study and their scores of valid links. In order to analyze concept maps, the researcher used one method of analysis by categorizing the level of connectivity and the number of valid links in each concept map. There were two kind of links in the concept maps. For example, when the student connects two unrelated topics such as 'adding rational expressions and multiplying rational expressions', then it is coded as 'invalid link'. On the other hand, when the student connects two related topics such as 'adding and subtracting polynomials", then that is coded as a "valid link'. Different concept maps included different number of valid links from 0 to more than 22 . All concept maps were categorized between low, medium, and high connectivity levels as shown in Table 2.

A total number of 390 students' concept maps from 130 students were examined. The majority of students showed progress regarding the connectivity levels of their maps (see Tables 3 and 4). The progress was observed through studying all the concept maps (390) created by the students (130) over the course of the study, their level of connectivity (Table 3), and the corresponding frequency of the number of students (Table 4) at each stage. Considering the median level of connectivity, we can see (Table 3) that students' connectivity codes and the number of valid links were low at Stage 0 but improved to 'Medium Connectivity' at Stages 2 and 3. 
Table 3. Median level of connectivity for all students at each stage

\begin{tabular}{lccc}
\hline Stage Number & Median Number of Valid Links & Median Using Connectivity Codes & Name of code \\
\hline Stage 0 & 10 & 1.0 & Low Connectivity \\
Stage 2 & 18 & 2.0 & Medium Connectivity \\
Stage 3 & 21 & 2.0 & Medium Connectivity \\
\hline
\end{tabular}

Table 4. Frequency of the number of students at each stage of the course

\begin{tabular}{lccc}
\hline Frequency at & Stage 0 & Stage 2 & Stage 3 \\
\hline Low Connectivity & 98 & 38 & 16 \\
Medium Connectivity & 20 & 69 & 75 \\
High Connectivity & 12 & 23 & 39 \\
\hline
\end{tabular}

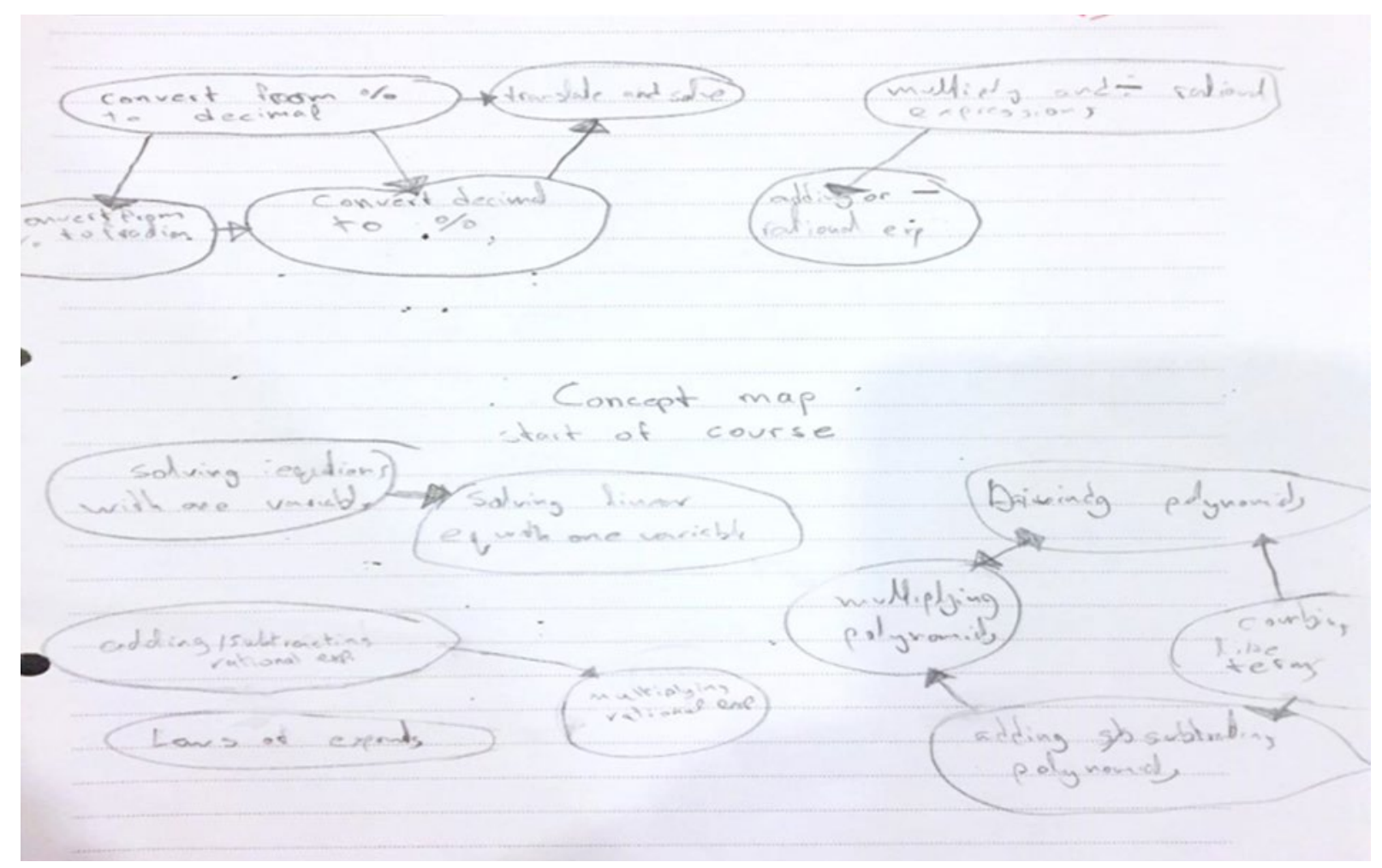

Figure 2. Student-1 first concept map (Low Connectivity)

Considering the frequency of students who displayed low, medium or high connectivity at different stages, we can see (Table 4) gradual improvement in students' connectivity from Stage 0 to Stage 3. The vast majority of students (98 out of 130) demonstrated low connectivity at Stage 0 but then this changed at Stage 2 and Stage 3, where the corresponding numbers became higher for the medium and high connectivity with the most impressive change showing a drop in the number of low connectivity from Stage 0 to Stage 3 by 82 (63\% of the students) (Table 4). The students started to see proper links between topics at Stages 2 and 3 indicating increased conceptual understanding.

The three concept maps that are presented below in Figure 2 are examples from one student, who demonstrated progression from low to high connectivity, to show how this progression looked on their concept maps collected at the three stages of the study.

At Stage 0 , in his first concept map, Student 1 created five different clumped groups of topics (Figure 2). He connected all topics related to polynomials together but not to other topics. He seemed to see individual distinct areas. Also, he showed 3 invalid links. For example, he connected solving linear equations with one variable to solving equations with one variable because it is about the word 'solving'. This shows that he did not understand that they represent the same objective. This map showed a total of 13 valid links -3 invalid links $=10$ links and connectivity code 1 (Low Connectivity). This also showed that the student missed opportunities to connect topics together and might have connected them procedurally more than conceptually following their topic titles (common words) only.

At Stage 1, in his second concept map (Figure 3), Student 1 connected topics conceptually more than procedurally, achieving 'Medium Connectivity'. For example, he created a net of topics and not clumps of groups as he had done in Stage 0. He created a central topic to which all of the other topics connected (Solving algebraic equations). In contrast, it is noticeable that he 


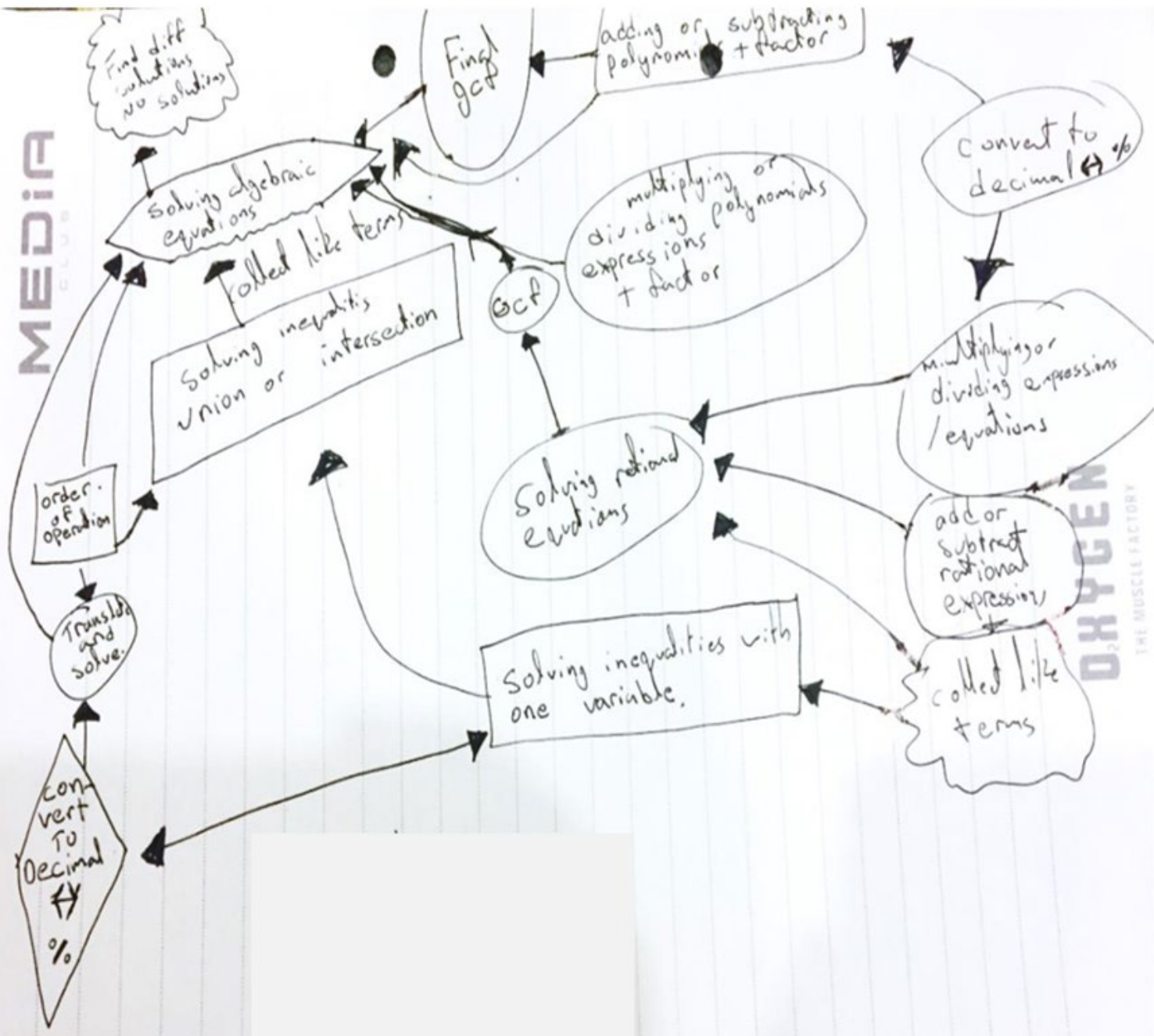

Figure 3. Student-1 second concept map (Medium Connectivity)

combined some objectives without clear ideas or reasons to combine them. For example, he combined multiplying or dividing polynomials expressions + factor. He is not seeing the very clear links between certain topics, but he does see more links than he did in Stage 0 . He showed a total of 22 - 2 invalid links $=20$ links and code 2 (Medium Connectivity). This students' map showed more connectivity and conceptual understanding than his level of understanding at Stage 0 . This indicates that he improved his conceptual understanding from Stages 0 to 1.

At Stage 2, in his third concept map Figure 4, Student 1 demonstrated a higher level of connectivity between the topics at Stage 3. He created a central important objective 'solving algebraic equations' and connected many other topics to it. He also showed more organized thoughts when he used 'combining like terms' and/or 'GCF' more than one time as needed. He showed a total of 34 valid links and code 3 (High Connectivity). The analysis of this student's maps showed improved conceptual understanding from one stage to another and significant progress during the course in his conceptual understanding.

Researcher's notes showed that students' confidence in creating and discussing the concept maps increased gradually during the course. The number of students who hesitated to start working on their maps or showed confusion while designing or discussing their maps were high at the beginning (i.e., 108 students in Stage 0 and 54 in Stage 2) but then became smaller towards the end (11 at Stage 3). She also noticed that the number of students who showed conceptual understanding was very limited at the start of the course and gradually improved towards the end of the course to include most of the students.

\section{Semi-structured Interviews; Qualitatively; Supporting Results}

In the interviews, the students expressed their opinions on the use of concept maps in their mathematics lessons. The students explained in their interviews that using concept maps has been a new experience that they were never exposed to during their previous academic years. They realized and appreciated 


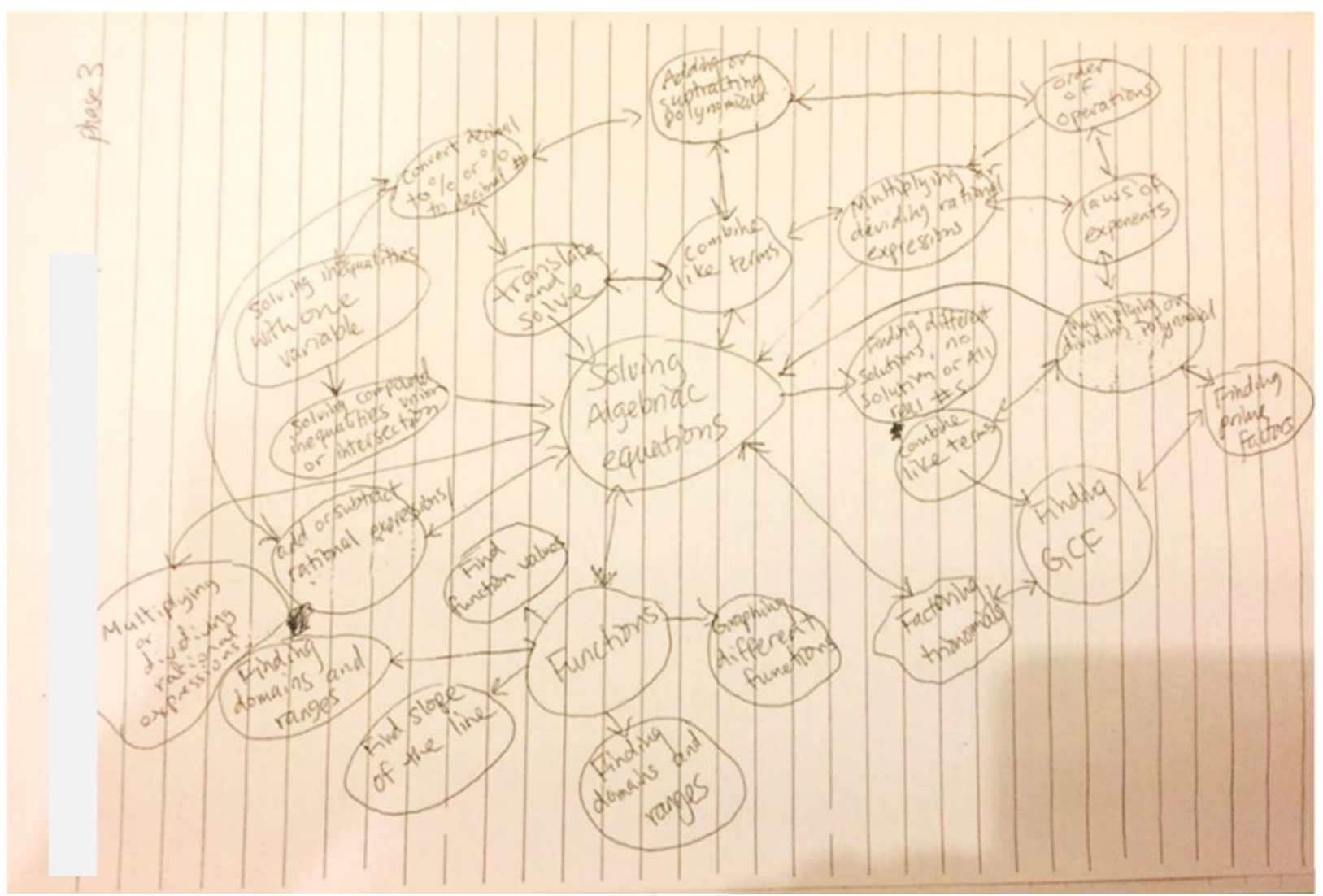

Figure 4. Student-1 third concept map (High Connectivity)

the importance of using concept maps in class and described how they influenced the way they think about learning mathematics and their understanding, as well as the way of working on everyday tasks and their motivation to do mathematics. The following are some samples of what the students said about:

- Increasing the time for thinking and using thinking skills to build relationships (relational understanding) and enhance understanding:

- "I was never exposed to concept maps before. I have been studying mathematics all my life and we just learned how to solve different problems and never how to relate topics we learn to each other. You gave us time to think and try to relate them and connect them" (Student 2)

- "Using these maps improved my understanding because I did not even know many mathematics terms. Now I know what is a rational equation means and how it is related to solving." (Student 3)

- Being involved in thinking about their own learning (metacognitive thinking):

- "I can see the huge difference between my map at the start and end of the course. It's very interesting how I understand the relation between them now. looking at the whole picture, it makes so much sense. I feel that I can understand maths well" (Student 2)

- "I never saw those maps in my life before. Looking at the first and last maps, they don't relate to each other. I didn't know or understand the concepts yet. My ideas were scattered in the first one. I did not realize the relations between those topics when we started the course. Dividing us into groups and allowing us to discuss the possible different relations between those topics after submitting the maps, made me realize the relations between them." (Student 3)

- Changing the way of working on everyday tasks and their motivation to do mathematics:

- "I loved using concept maps although I never experienced it in the past. I used to find maths very difficult to understand. Using concept maps was different and interesting. I remember one time we were playing a speed game, you asked us to solve one hard quadratic equation using factoring. I remembered the meaning of solve from the concept map we discussed in class and thought I could use the quadratic formula to solve in a faster way instead of factoring and compare the answers, and the answers matched from factoring and the quadratic formula. I remember how proud you were of me and you showed the class how I related solving quadratic equations using different methods as we learned from the concept maps. I used to hate maths but now it's becoming easier and making so much sense. I can do it"(Student 4)

- "Also, learning the relations between topics every time we take a new lesson shows me that I can also 
Table 5. Test scores and percent increase/decrease at all stages of the course

\begin{tabular}{lccccccccc}
\hline Group Number & G1 & G2 & G3 & G4 & G5 & G6 & G7 & G8 & $\begin{array}{c}\text { Mean for all } \\
\text { Groups Together }\end{array}$ \\
\hline Mean in Percentage at Stage 0 & 48 & 41 & 46 & 46 & 30.6 & 28 & 39.5 & 27 & 38.6 \\
Mean in Percentage at Stage 1 & 74 & 67 & 62 & 73.5 & 63.5 & 67.3 & 75 & 58 & 67.6 \\
Percentage Increase/Decrease from Stage 0 to 1 & 54.2 & 63.4 & 34.8 & 59.8 & 107.5 & 140.4 & 89.9 & 114.8 & 83.1 \\
Mean in Percentage at Stage 2 & 87.8 & 69.3 & 71.8 & 73.9 & 76.3 & 74.5 & 79.5 & 64 & 74.7 \\
Percentage Increase/Decrease from Stage 1 to 2 & 18.7 & 3.4 & 15.8 & 0.5 & 20.2 & 10.7 & 6 & 10.3 & 10.7 \\
Mean in Percentage at Stage 3 & 93.2 & 77.9 & 82.1 & 82.3 & 78.2 & 79.1 & 79.8 & 75.1 & 81.3 \\
Percentage Increase/Decrease from Stage 2 to 3 & 6.1 & 12.3 & 14.4 & 11.4 & 4.6 & 7.3 & -0.3 & 18.2 & 8.8 \\
\hline
\end{tabular}

Table 6. Procedural and conceptual average success rate in all tests in the course

\begin{tabular}{|c|c|c|c|c|c|c|c|c|}
\hline \multicolumn{9}{|c|}{ Average Success Rate in } \\
\hline \multirow{2}{*}{$\begin{array}{l}\text { Group } \\
\text { Number }\end{array}$} & \multicolumn{2}{|c|}{ The Level Quiz-Stage 0} & \multicolumn{2}{|c|}{ Test 1 -Stage 1} & \multicolumn{2}{|c|}{ Test 2-Stage 2} & \multicolumn{2}{|c|}{ Test 3-Stage 3} \\
\hline & Procedural & Conceptual & Procedural & Conceptual & Procedural & Conceptual & Procedural & Conceptual \\
\hline G1 & 46.0 & 20.0 & 70.7 & 71.5 & 83.7 & 82.7 & 91.7 & 85.0 \\
\hline G2 & 37.8 & 16.5 & 69.7 & 71.4 & 80.7 & 77.1 & 83.9 & 81.2 \\
\hline G3 & 45.1 & 21.6 & 61.3 & 57.8 & 78.1 & 76.3 & 100.0 & 95.4 \\
\hline G4 & 40.0 & 14.0 & 68.8 & 75.3 & 87.1 & 85.4 & 85.3 & 83.5 \\
\hline G5 & 31.4 & 4.9 & 60.5 & 66.9 & 81.6 & 78.6 & 82.1 & 79.5 \\
\hline G6 & 27.0 & 6.0 & 61.0 & 64.1 & 78.0 & 75.0 & 79.9 & 78.8 \\
\hline G7 & 31.3 & 16.7 & 67.1 & 73.4 & 83.8 & 81.5 & 81.7 & 81.3 \\
\hline G8 & 26.0 & 14.4 & 39.0 & 44.2 & 78.6 & 72.1 & 79.1 & 77.5 \\
\hline $\begin{array}{l}\text { Average of } \\
\text { All Groups }\end{array}$ & 35.6 & 14.3 & 62.3 & 65.6 & 81.5 & 78.6 & 85.5 & 82.8 \\
\hline
\end{tabular}

understand and see that most concepts can lead to solving algebraic equations." (Student 3)

- "I am usually easy to feel bored in class. At the start of the course, I felt that it was weird that we had to draw those maps. But, the more we learned about those maps, discussed them, and understood the relations between topics, the more fun it became.... I never knew that adding and subtracting polynomials can lead to solving them, and can involve different operations and applications. This is very interesting and seems like a puzzle that we need to connect its pieces together. I would like to find out how to connect new topics in future maths courses to our current topics"(Student 5).

The above words of students also highlight the role of discussions and their reflection on their own students' maps from the start to the end of the course. These reflections were promoted by the teacher and her use of concept maps, and emphasized to the students the relations between objectives during the course.

\section{Level Quiz and Test Scores; Quantitatively; Supporting Results}

This section presents the detailed data and scores of the level quiz and all the major tests completed during the course. The researcher aimed to show the changes in students' scores at every test in order to reflect the changes in students' mathematics understanding. Table 5 shows the test scores for the students at all stages of the study.

Table 5 demonstrates that the students' test scores noticeably and gradually improved during the stages of the course. This might be an indicator of a noticeable improvement that occurred in the students' mathematics knowledge during the course.

\section{Procedural and Conceptual Responses; Quantitatively; Supporting Results}

Table 6 reflects the average success rate for procedural and conceptual questions in each test. The success rate is defined as students gaining $70 \%$ or more of the marks for procedural or conceptual questions in each test.

The results in Table 6 show that the success rates at the start of the course were higher for questions required procedural rather than conceptual thinking. This suggests that students, at the start of the course, were more confident and prepared to deal with situations involving procedural rather than conceptual understanding. The table also shows a gradual improvement in student's mathematical understanding and a noticeable increase in the success rates for both types of questions during the course. Moreover, the table shows an impressive difference in success concerning conceptual understanding from the level quiz to the first test. This can be a stronger indicator of students' improvement in their mathematics understanding during the course. 
Table 7. Success rates for similar questions between from the Level quiz to Test 3

\begin{tabular}{|c|c|c|c|c|c|c|c|}
\hline Questions in Level Quiz & $\begin{array}{l}\text { Procedural } \\
\text { or } \\
\text { Conceptual }\end{array}$ & $\begin{array}{c}\text { Number of } \\
\text { Students at } \\
\text { Success Rate / } \\
130 \\
\end{array}$ & $\%$ & Questions in Test 3 & $\begin{array}{l}\text { Procedural } \\
\text { or } \\
\text { Conceptual }\end{array}$ & $\begin{array}{c}\text { Number of } \\
\text { Students at } \\
\text { Success Rate / } \\
130 \\
\end{array}$ & $\%$ \\
\hline $\begin{array}{l}\text { 1-b) Simplify Using } \\
\text { Exponential Properties }\end{array}$ & Procedural & 12 & 9.2 & $\begin{array}{l}\text { Divide and simplify } \\
\text { rational and exponential } \\
\text { expressions }\end{array}$ & Procedural & 106 & 84.8 \\
\hline $\begin{array}{l}\text { 3- Solve Linear Equation } \\
\text { with One Variable }\end{array}$ & Procedural & 65 & 50.0 & $\begin{array}{l}\text { 1) Solve linear equation } \\
\text { with one variable }\end{array}$ & Procedural & 124 & 99.2 \\
\hline $\begin{array}{l}\text { 5- Translate the meaning } \\
\text { of equilibrium into an } \\
\text { equation and solve } \\
\text { (word problem) }\end{array}$ & Conceptual & 15 & 11.5 & $\begin{array}{l}\text { 12) Find the break-even } \\
\text { point and solve the } \\
\text { quadratic equation } \\
\text { (word problem) }\end{array}$ & Conceptual & 102 & 81.6 \\
\hline
\end{tabular}

Table 8. Pass-Fail rates for each participating group in the study

\begin{tabular}{lcccc}
\hline Group Number & Pass & Not Pass & Pass (percentage) & Not Pass (percentage) \\
\hline 1 & 10 & 0 & 100 & 0.0 \\
2 & 16 & 3 & 84.2 & 15.8 \\
3 & 15 & 2 & 88.2 & 11.8 \\
4 & 18 & 2 & 90.0 & 10.0 \\
5 & 12 & 2 & 85.7 & 14.3 \\
6 & 16 & 2 & 88.9 & 11.1 \\
7 & 17 & 2 & 89.5 & 10.5 \\
8 & 11 & 2 & 84.6 & 15.4 \\
\hline Total No / Average (\%) & 115 & 15 & 88.9 & 11.1 \\
\hline
\end{tabular}

Moreover, the researcher considered studying similar questions from the level quiz and Test 3. Table 7 compares the success rates for those questions from the level quiz to Test 3 .

The results from the level quiz and Test 3 showed that the students improved their abilities to solve procedural and conceptual problems in these topic areas during the course. Considering the similar conceptual questions, the students showed a percent increase of $609 \%$ in their success, which might be another indicator that using concept maps has reflected on students' conceptual understanding from the start to the end of the course.

\section{Students' Performance and Success Rates; Quantitatively: Supporting Results}

This section presents the success rate for each participating group included in the study as shown in Table 8 . The table also compares the average success rates between the students of this study and the students of MFU in Math 096 as shown in Figure 5. The comparison shows that the pass rate for the students in the study is higher than the pass rate in MFU as a whole. This is another indicator that concept maps might have positively influenced students' mathematical understanding and achievement in the study compared to MFU students who were not exposed to concept maps.

\section{DISCUSSION}

This study examined the use of concept maps in mathematics learning in a foundation course at a
University in Kuwait. The data analyzed at the start of the course for all the 130 students participating in the study showed low scores in both mathematical achievement as shown in the level quiz scores, and mathematical understanding as shown in the level quiz (procedural/conceptual) analysis. The data also showed that they had low connectivity in their concept maps analysis as they scored at 'Low Connectivity' at the start of the course. In addition, concept mapping provided a theoretical model that explored the students' mental activities when engaging new and old concepts together (Kinchin et al., 2000). The participating students had graduated from school with a weak conceptual view of mathematical topics which suggested that they had weak conceptual understanding. They appeared to be more familiar with working procedurally and therefore having procedural understanding rather than conceptual understanding at the start of the course, which confirms findings from previous studies (e.g., McGowen \& Tall, 1999). Similarly, with McGowen and Tall (1999), low achieving students in this study at the start of the course were unable to develop mastery skills or any degree of proficiency with the material they had already seen previously in their mathematics lessons. They lacked what Skemp (1976) called relational understanding; understanding that requires higherorder thinking skills to conceptualize and build higherorder relationships and higher-order concepts (Skemp, 1986). This means, most of the students participating in the study joined the course with weak mathematical knowledge and understanding. 


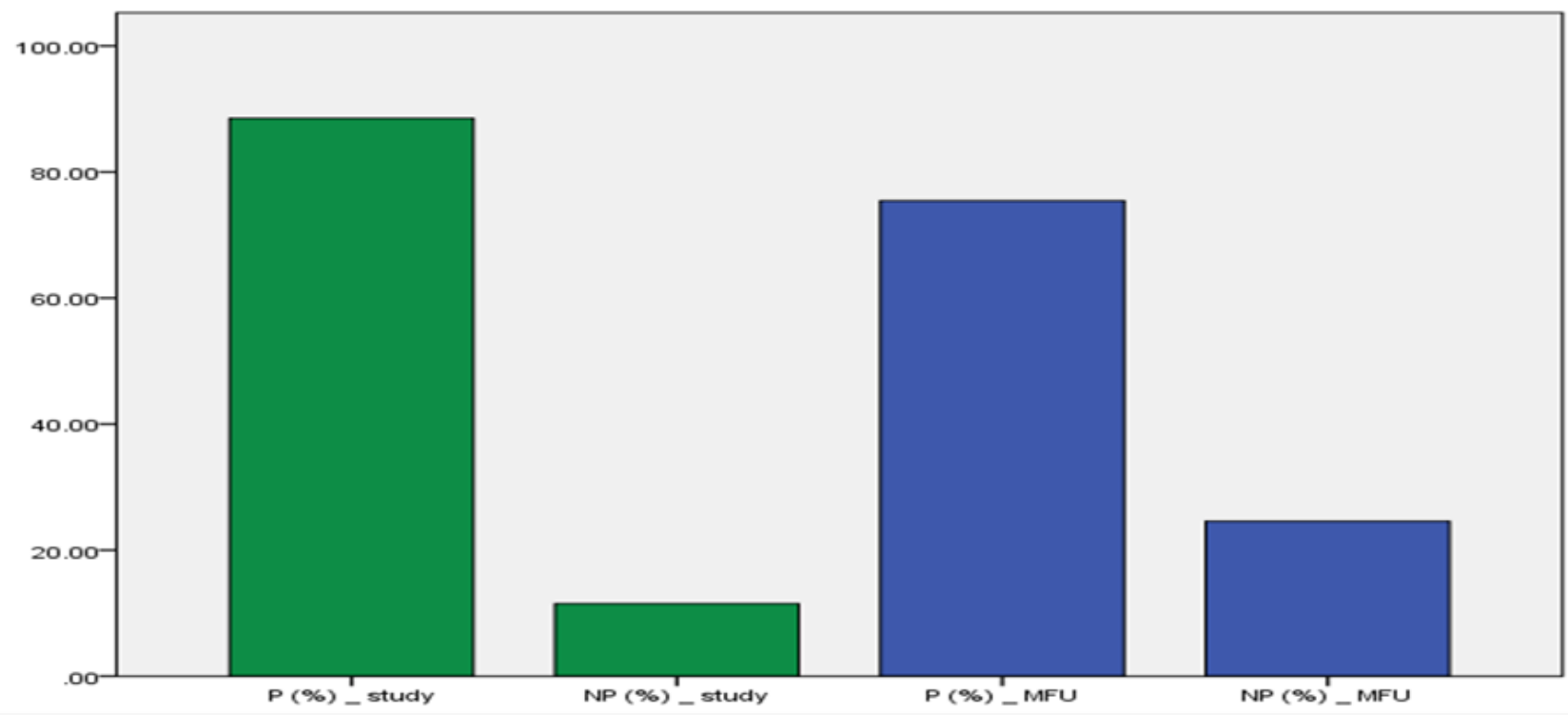

Figure 5. A comparison in pass-fail rates for Math 096 between students participating in the study and other Math 096 students in MFU. (P=Pass and NP= Not Pass)

Using concept maps in the class created an engagement within the learning process. This enabled the students to adopt and create a diverse perspective of methods for solving problems and learn that if they have selected the best links between objectives, why, or how can those objectives be linked, which promoted higherorder thinking (Smith, 2014). Lewis and Smith (1993) suggested that higher-order thinking "occurs when a person takes new information and information stored in memory and interrelates and/or rearranges and extends this information to achieve a purpose or find possible answers in perplexing situations" (1993, p. 32). The higher-order thinking skills were developed in this research as a result of the inclusion of the concept maps and the follow-up discussions, which encouraged students to reflect and talk about their maps. By working on their concept maps, students needed to use their higher-order thinking skills to analyze, evaluate, and create knowledge (Apino \& Retnawati, 2017; Liu, 2009). By creating their concept maps, analysing them, studying why and how to connect concepts with each other, the students in this study learned to organize their mathematical ideas in a conceptual schema, which improved their conceptual understanding as supported by Akkaya et al. (2005). This was also reflected on the researcher's journal, where a gradual decrease in the number of confused students was recorded followed by an increase in the number of students demonstrating conceptual understanding. This improvement in their conceptual understanding became apparent as students' concept maps evolved, which adds more evidence to what previous research found regarding the benefits of using concept maps to study students' mathematical understanding (e.g., Cliburn, 1990; Lambiotte \& Dansereau, 1991; Lanier, 1997; Laturno, 1993; McGowen \& Tall, 1999; McGowen \& Davis, 2019; Moreira, 1979;
Novak, 1990; Park \& Travers, 1996; Skemp, 1986; Williams, 1998). In addition, during the course, the students were exposed to group discussions where they explored and built the different relations between the concepts. Also, the teacher talked about the relationships present between different concepts while teaching individual lessons. Using concept maps in this course helped to develop a meaningful and rich experience for the students by encouraging them to relate the new ideas that they were learning to their existing ideas that they had learned in the past. The teacher had an important role in engaging the students in higher-order thinking skills (Henningsen \& Stein, 1997). This included choosing the right task for the students such as using concept maps, providing teacher's support and actions such as scaffolding and continuous requests to provide explanations of how to show meaningful connections and explanations while solving different problems (Henningsen \& Stein, 1997). In addition, the researcher in this study used concept mapping as a self-assessment so that students can see their misconceptions, which was shown to improve students' understanding as supported by Kazemi and Stipek (2001), and Swan (2005). Concept mapping was a relational instrument that could outline relationships between ideas and associate elements spontaneously in this study as supported by Davies (2010).

In this study, the students were able to discuss their concept maps in groups after submitting them to the teacher. They were able to use discussions and test ideas, receive and provide ideas to each other, put together their ideas into words, communicate mathematically, be involved in mathematical arguments, make new claims, and construct a deeper understanding of concepts (Davies, 2011; Francisco, 2013; McGowen \& Tall, 1999; Williams, 1998). The students showed significant 
improvement in their test scores, on both procedural and conceptual tests, with special attention to their conceptual tests. This showed an expected improvement in students' achievement towards the end of a mathematics foundation course, which might relate to long-term benefits in learning. In addition, the students reflected a higher success rate and improvement compared to the rest of the department's success rate for students who did not participate in the study. Also, their connectivity level improved to 'Medium Connectivity'. This showed an increased ability in the students to produce concept maps with higher connectivity which suggests an increased ability for those students to understand mathematics conceptually and see the subject as a connected whole (Skemp, 1986). Also, the students who participated in the study, gained higherorder thinking skills to conceptualize and built higherorder relationships (schemata) and higher-order concepts (Skemp, 1986). This is a valid indicator of the increase in students' conceptual understanding as the students started to see valid links between topics at earlier stages as of the first month of the course. In their interviews, the students explained that the use of concept maps had improved their conceptual understanding and motivation towards studying mathematics in the future. This might be an indicator that the students in the study had improved their mathematical understanding during the course especially their conceptual understanding.

The students continued to improve their test scores and their conceptual understanding through the procedural/conceptual tests, and their overall average towards the end of the course. This also showed that concept mapping can be used as a monitoring and promoting assessment of their conceptual and mathematical understanding progress during the course as recommended by Kinchin et al. (2000).

Lastly, the researcher in this study tried to overcome the disadvantages mentioned by Davies (2011) as follows: 1) at the start of the course, the teacher used one example to show how to relate some objectives to each other before asking them to draw their first mathematics concept maps. She used unrelated objectives to the objectives used in the study to avoid bias of leading the students into copying her explained concept maps. She showed them the possibilities of using a net, chain, or spoke as recommended by Kinchin et al. (2000). The students were given the option to use the design they wished to use. This could overcome the idiosyncratic in terms of design, 2) the teacher encouraged the students to express their mathematical understanding through the links they were requested to draw, without the need to memorize the links and relation between elements from one stage to another, 3 ) the students were always allowed to asked questions during the course related to concept mapping especially when were provided with the science concept mapping example at the start of the course. They did not show any frustration or overwhelming feelings. They showed noticeable motivation to draw their concept maps at later stages.

\section{LIMITATIONS AND RECOMMENDATIONS}

The sample size for the students participating in this study was 130 students. Studying a larger number of students might produce better and more generalizable results. Further research is recommended on the use of concept maps in the classroom as a teaching tool with a greater sample size of participants and to test it at the high school levels or higher college-level courses.

The role of the researcher-teacher is often considered a limitation as a potential for bias. To overcome this limitation and avoid biases in this study, the researcher kept a research journal not just for recording details of events, but also for checking the strengths and weaknesses of the study and her own beliefs and positionality, in other words, as an instrument for reflexivity (Berger, 2015; Watt, 2007). She shared her research journal comments with her supervisors in order to use reflexivity during the study and design the educational approaches needed without changing the course standards. Also, the researcher was not involved when assigning any of her classes, designing the syllabus and the grading scheme of the course, and designing tests as they were designed by a testing committee that she was not involved in. Lastly, the Student Information Sheet explained all the ethical information and the aims of the study and showed the clearance of their voluntarily participation without any pressure on their student-teacher relation. Further research is recommended on the use of concept maps and involving different teachers with different teaching styles in future studies in order to compare different teachers' styles and their influence on students' understanding.

\section{CONCLUSION}

There has been a paucity during the last 20 years in research concerning the use of concept maps in mathematics teaching and learning despite the welldocumented evidence reported in the past, mainly before the '90s. This study makes a contribution towards addressing this issue. The study investigated the influence of using concept maps in mathematics learning, particularly conceptual understanding and how this could be monitored and enhanced while studying a foundation course. The students' conceptual understanding was shown to be weak at the start of course as has been identified by the researcher and reported by other mathematics educators. The researcher aimed to improve students' mathematical understanding through the use of concept maps. Also, concept maps were used as a monitoring and important assessment tool to improve and promote students' 
conceptual understanding during the course. The students showed noticeable improvements in their mathematical understanding especially their conceptual understanding. This was shown by using students' test scores, concept maps analysis, different testing styles (procedural/conceptual), and overall success rate measurements during the course. The study showed that concept maps can be successful as a tool for assessing, monitoring, and promoting students' conceptual understanding for a college-level course. This study recommends further research to examine more systematically the possible educational merits of using concept maps within mathematics classes and longitudinal studies to determine whether and to what extent their use can predict academic achievement and success.

\section{REFERENCES}

Akkaya, R., Karakirik, E., \& Durmus, S. (2005). A Computer Assessment Tool for Concept Mapping. Turkish Online Journal of Educational TechnologyTOJET, 4(3), 3-6.

Apino, E., \& Retnawati, H. (2017). Developing instructional design to improve mathematical higher order thinking skills of students. In Journal of Physics: Conference Series (Vol. 812, No. 1, p. 012100). IOP Publishing. https:// doi.org/10.1088/ 1742-6596/812/1/012100

Baroody, A. J. (2003). The development of adaptive expertise and flexibility: The integration of conceptual and procedural knowledge. In A. J. Baroody \& A. Dowker (Eds.), The development of arithmetic concepts and skills: Constructing adaptive expertise (pp. 1-33). Mahwah, NJ: Erlbaum.

Berger, R. (2015). Now I see it, now I don't: Researcher's position and reflexivity in qualitative research. Qualitative Research, 15(2), 219-234. https:// doi.org/10.1177/1468794112468475

Cliburn, J. W. (1990). Concept maps to promote meaningful learning. Journal of College Science Teaching, 19, 212-217.

Cohen, L., Manion, L., \& Morrison, K. (2013). Research Methods in Education. London: Routledge. https://doi.org/10.4324/9780203720967

Davies, M. (2011). Concept mapping, mind mapping and argument mapping: what are the differences and do they matter?. Higher education, 62(3), 279-301. https:// doi.org/10.1007/s10734-010-9387-6

DeCaro, M. S. (2016). Inducing mental set constrains procedural flexibility and conceptual understanding in mathematics. Memory $\mathcal{E}$ cognition, 44(7), 1138-1148. https:// doi.org/10.3758/s13421016-0614-y

Driver, R. (1981) Pupils' alternative framework in science. European Journal of Science Education, 3(1),
93-101.

https:/ / doi.org/10.1080/0140528810030109

Ernest, P. (1991). The Philosophy of Mathematics Education. London: Routledge.

Ernest, P. (2011). The Psychology of Learning Mathematics: The Cognitive, Affective and Contextual Domains of Mathematics Education. Saarbrücken, LAP Lambert Academic Publ.

Francisco, J. M. (2013). Learning in collaborative settings: Students building on each other's ideas to promote their mathematical understanding. Educational Studies in Mathematics, 82(3), 417-438. https:/ / doi.org/10.1007/ s10649-012-9437-3

Gray, E. M., \& Tall, D. O. (1994). Duality, ambiguity, and flexibility: A "proceptual" view of simple arithmetic. Journal for research in Mathematics Education, 25(2), 116-140. https:/ / doi.org/10.2307/ 749505

Heinze, A., Star, J. R., \& Verschaffel, L. (2009). Flexible and adaptive use of strategies and representations in mathematics education. ZDM Mathematics Education, 41, 535-540. https://doi.org/10.1007/ s11858-0090214-4

Henningsen, M., \& Stein, M. K. (1997). Mathematical tasks and student cognition: Classroom-based factors that support and inhibit high-level mathematical thinking and reasoning. Journal for research in mathematics education, 28(5), 524-549. https:/ / doi.org/10.2307/749690

Hiebert, J., \& Carpenter, T. P. (1992). Learning and teaching with understanding. In D. A. Grouws (Ed.), Handbook of research on mathematics teaching and learning: A project of the National Council of Teachers of Mathematics (pp. 65-97). Macmillan Publishing Co, Inc.

Hiebert, J., \& Lefevre, P. (1986). Conceptual and procedural knowledge in mathematics: An introductory analysis. In J. Hiebert (Ed.), Conceptual and procedural knowledge: The case of mathematics (pp. 1-27). Hillsdale, NJ: Lawrence Erlbaum Associates.

Jonassen, D. H., Reeves, T. C., Hong, N., Harvey, D., \& Peters, K. (1997). Concept mapping as cognitive learning and assessment tools. Journal of interactive learning research, 8(3), 289.

Kadijevich, D., \& Haapasalo, L. (2001). Linking procedural and conceptual mathematical knowledge through CAL. Journal of Computer Assisted Learning, 17(2), 156-165. https:// doi.org/10.1046/j.0266-4909.2001.00168.x

Kazemi, E., \& Stipek, D. (2001). Promoting conceptual thinking in four upper-elementary mathematics classrooms. Elementary School Journal, 102, 59-80. https://doi.org/10.1086/499693 
Kelly, G. A. (1955). The Psychology of Personal Constructs: Volume One: Theory and Personality. London: Routledge.

Kinchin, I. M., Hay, D. B., \& Adams, A. (2000). How a qualitative approach to concept map analysis can be used to aid learning by illustrating patterns of conceptual development. Educational research, 42(1), 43-57. https:/ / doi.org/10.1080/001318800363908

Lambiotte, J. G., \& Dansereau, D. F. (1991). Effects of knowledge maps and prior knowledge on recall of science lecture content. The Journal of Experimental Education, 60(3), 189-201. https:/ / doi.org/10.1080/ 00220973.1992.9943875

Lanier, P. (1997). Assesment in the Service of Instruction [Paper presentation]. Annual meeting of the National of Supervisors of Mathematics, St.Paul, MN.

Laturno, J. (1993). The validity of concept maps as a research tool in remedial college mathematics. Proceedings of the 16th Annual Meeting of the Group for the Psychology of Mathematics Education, North American Chapter (pp. 60-66). Baton Rouge, LA.

Lewis, A., \& Smith, D. (1993). Defining higher order thinking. Theory into practice, 32(3), 131-137. https:/ / doi.org/10.1080/00405849309543588

Liu, X. (2009). Essentials of science classroom assessment. Sage Publications. https://doi.org/10.4135/ 9781483349442

McGowen, M. A., \& Davis, G. E. (2019). Spectral analysis of concept maps of high and low gain undergraduate mathematics students. The Journal of Mathematical Behavior, 55, 100686. https:/ / doi.org/ 10.1016/j.jmathb.2019.01.002

McGowen, M., \& Tall, D. (1999). Concept Maps \& Schematic Diagrams as Devices for Documenting the Growth of Mathematical Knowledge. The 23rd International Group for the Psychology of Mathematics Education (Vol. 3). Haifa, Israel.

Michener, E. R. (1978). Understanding understanding mathematics. Cognitive science, 2(4), 361-383. https://doi.org/10.1207/s15516709cog0204_3

Miller, S. P., \& Hudson, P. J. (2007). Using evidencebased practices to build mathematics competence related to conceptual, procedural, and declarative knowledge. Learning Disabilities Research \& Practice, 22(1), 47-57. https://doi.org/10.1111/j.15405826.2007.00230.x

Moreira, M. A. (1979). Concept Maps as Tools for Teaching. Journal of College Science Teaching, 8(5), 283-86.

Novak, J. D. (1990). Concept mapping: A useful tool for science education. Journal of research in science teaching, 27(10), 937-949. https://doi.org/10.1002/ tea.3660271003
Park, K. \& Travers, K. (1996). A Comparative Study of a Computer-Based and a Standard College First-Year Calculus Course. In E.Dubinsky, A. Schoenfeld, \& J. Kaput (Eds.), Research in Collegiate Mathematics Education (pp. 155-176). Providence, RI: American Mathematical Society. https://doi.org/10.1090/ cbmath/006/07

Peled, I., \& Segalis, B. (2005). It's not too late to conceptualize: Constructing a generalized subtraction schema by abstracting and connecting procedures. Mathematical Thinking and Learning, 7(3), 207-230. https://doi.org/10.1207/s15327833 mt10703_2

Pfund, H., \& Duit, R. (1994) Bibliography - Students' Alternative Frameworks and Science Education (4th edn). Kiel: Institute for Science Education.

Piaget, J., \& Cook, M. (1952). The origins of intelligence in children (Vol. 8, No. 5, p. 18). New York: International Universities Press. https://doi.org/ 10.1037/11494-000

Reinfried, S. (2006). Conceptual change in Physical Geography and Environmental Sciences through mental model building: The example of groundwater. International Research in Geographical and Environmental Education, 15(1), 41-61. https:/ / doi.org/10.2167/irgee186.0

Rittle-Johnson, B., \& Alibali, M. W. (1999). Conceptual and procedural knowledge of mathematics: Does one lead to the other? Journal of Educational Psychology, 91(1), 175-189. https://doi.org/10.1037 /0022-0663.91.1.175

Rumelhart, D. E., \& Norman, D. A. (1978). Accretion, tuning, and restructuring: Three modes of learning. In J. W. Cotton \& R. Klatzky (Eds.), Semantic factors in cognition (pp. 37-53). Hillsdale, NJ: Lawrence Erlbaum.

Schneider, M., Rittle-Johnson, B., \& Star, J. R. (2011). Relations among conceptual knowledge, procedural knowledge, and procedural flexibility in two samples differing in prior knowledge. Developmental Psychology, 47, 1525-1538. https:// doi.org/10.1037/a0024997

Sfard, A. (1991). On the dual nature of mathematical conceptions: Reflections on processes and objects as different sides of the same coin. Educational studies in mathematics, 22(1), 1-36. https://doi.org/ $10.1007 /$ BF00302715

Siegler, R. S., \& Crowley, K. (1994). Constraints on learning in nonprivileged domains. Cognitive Psychology, 27, 194-226. https://doi.org/10.1006/ cogp.1994.1016

Skemp, R. (1976). Instrumental understanding and relational understanding. Mathematics Teaching, 77, 20-26. 
Skemp, R. R. (1979). Intelligence, Learning, and Action. a New Model for Theory and Practice in Education: New York Wiley.

Skemp, R.R. (1986). The Psychology of Learning Mathematics. London: Penguin Books

Smith, R. O. (2014). Beyond passive learning: Problembased learning and concept maps to promote basic and higher-order thinking in basic skills instruction. Journal of Research and Practice for Adult Literacy, Secondary, and Basic Education, 3(2), 50-55.

Soliman, M., \& Hilal, A. (2016). Investigating the effects of Computer-Assisted Instruction on Achievement and Attitudes towards Mathematics Among Seventh-Grade Students in Kuwait. International Journal of Technology in Mathematics Education, 23(4), 145-160.

Swan, M. (2005). Standards Unit-Improving learning in mathematics: challenges and strategies. DfES. Retrieved from http://www.Ncetm.org.uk/files/ 224/improving_learning_in_mathematicsi.pdf

Tall, D. (1994b). Understanding the processes of advanced mathematical thinking [Invited ICMI lecture]. International Congress of Mathematicians, Zurich.

Thomas, G. (2011). A typology for the case study in social science following a review of definition, discourse, and structure. Qualitative inquiry, 17(6), 511-521. https:/ / doi.org/10.1177/1077800411409884
Verschaffel, L., Luwel, K., Torbeyns, J., \& Van Dooren, W. (2009). Conceptualizing, investigating, and enhancing adaptive expertise in elementary mathematics education. European Journal of Psychology of Education, 24, 335-359. https: / / doi.org/10.1007/BF03174765

Von Glasersfeld, E. (1998). Cognition, construction of knowledge, and teaching. In Constructivism in science education (pp. 11-30). Springer, Dordrecht. https://doi.org/10.1007/978-94-011-5032-3_2

Vygotsky, L. S. (1978). Mind in Society. Cambridge: Harvard University Press. In E. Wenger, N. White, \& J. Smith (2009), Digital habitats: Stewarding technology for communities. CP Square Press.

Watt, D. (2007). On becoming a qualitative researcher: the value of reflexivity. Qualitative Report, 12(1), 82101.

Wilkerson-Jerde, M. H., \& Wilensky, U. J. (2011). How do mathematicians learn math?: Resources and acts for constructing and understanding mathematics. Educational Studies in Mathematics, 78(1), 21. https:/ / doi.org/10.1007/s10649-011-9306-5

Williams, C. G. (1998). Using concept maps to assess conceptual knowledge of function. Journal for Research in Mathematics Education, 414-421. https:/ / doi.org/10.2307/749858 


\section{APPENDIX A}

\section{Mathematics Foundation Unit}

\section{Math 096-Level Quiz}

Student name: ID\# Section\#

1. Simplify:
a) $30-4\left[9 \div\left(5-2^{3}\right)\right]+(9-7)^{2}$
[4 marks]
b) $\frac{21 a^{4} b c}{7 a^{2} b^{3} c^{-2}}$
[4 marks]

2. Evaluate each of the following expression according to each given value of $x$ :

[4 marks]
a) $\frac{4}{x-1}($ when $x=1)$
b) $\frac{0}{x+3}($ when $x=5)$

3. Solve the equation below for $x$

[5 marks] $3(4 x-2)-1=2(3 x-1)+7$

4. The Smith family paid a total of $\$ 4000$ as a down payment on a new luxury recreational boat. If this represent $20 \%$ of the total price, calculate the price of the new boat.

[4 marks]

5. A computer company uses the polynomials $R=150 x^{2}+50 x-10$ and $C=150 x^{2}+20$ to estimate its monthly revenue $R$ and $\operatorname{cost} C$ in dollars for producing $x$ cell phones per month. Find the number of cell phones produced when the revenue and cost reach equilibrium. Explain your final answer.

[4 marks]

\section{http://www.ejmste.com}

